\title{
Finite Element Analysis of Vehicle Load Effect on Harvesting Energy Properties of a Piezoelectric Unit
}

\author{
Chunhua Sun, Hongbing Wang, Jie Liu, Guangqing Shang \\ Department of Mechanic and Electronic Engineering, Suzhou Vocational University, Suzhou, China \\ Email: chh sunny@163.com
}

Received 4 September 2015; accepted 27 September 2015; published 30 September 2015

Copyright (C) 2015 by authors and Scientific Research Publishing Inc.

This work is licensed under the Creative Commons Attribution International License (CC BY). http://creativecommons.org/licenses/by/4.0/

(c) (i) Open Access

\section{Abstract}

To realize the goal of harvesting energy from pavement vibration on a large scale, a new type of piezoelectric harvesting units as the energy transducer has been proposed. The piezoelectric harvesting units are paved $40 \mathrm{~mm}$ below the asphalt, which is the same as thickness of the top layer of typical asphalt pavement in China. The spacing distance is $2200 \mathbf{~ m m}$, which is the same as the one between two tires of a normal vehicle. A mathematical model of the unit is deduced on Meda empirical formula and Hamilton principle and piezoelectric equations. Effects of the external vehicle load on its harvesting energy properties and pavement deformation and stress are analyzed with the finite element method. The results show that the excited voltage is linearly variation with contact pressure while the harvested electrical energy exponential varies with contact pressure. The more the contact pressure is, the larger the harvested electrical energy and the deformation and stress are. The harvested electrical energy also increases with the load frequency. At least 100 $\mathrm{mJ}$ of electrical energy can be collected with the proposed piezoelectric harvesting unit. It shows that the technology application of the piezoelectric harvesting energy from pavement is promising.

\section{Keywords}

Pavement Vibration, Piezoelectric Harvesting Unit, Positive Piezoelectric Effect, Finite Element Analysis

\section{Introduction}

Vibration-based piezoelectric harvesting energy technology has received growing attention over the last decade. 
The main application of the technology is to power small electronic devices by using the vibration energy available in ambient environment, such as MEMS micro-power devices [1] [2]. Recently, there also has a breakthrough in large power generation by using the technology. According to the report [3], when a vehicle is driven along the road, the piezoelectric harvester embedded under the pavement is deformed and produces electric charge. Although one piezoelectric harvester can only produce a small amount of electricity, millions of the harvesters generate huge electrical power, which can be transported into the state grid for accumulating or storing it for later use. If the technology is widely applied, the vast road will become a large electricity farm for supplying human being with inexhaustible energy.

The technology of vibration-based piezoelectric energy harvesting from pavement has attracted more and more attention of academia and industry. It will be one important direction of piezoelectric harvesting energy by replacing conventional electricity generation. It also will be a groundbreaking systematic works. However, as a new concept, the state of studying the technology is still in its infancy, a complete theoretical system has not been established. Few researches have dealt with it until now. Patent on the piezoelectric method and system for harvesting vibration from road was invented [4]. Finite element analysis of Cymbal piezoelectric transducers for harvesting energy from asphalt pavement was done in Ref. [5]. Effects of structural parameters and the planted depth of the piezoelectric harvester on electromechanical properties and pavement deformation were studied via the finite element method (FEM), a new kind of piezoelectric harvesting unit was proposed [6] [7]. Based on this structure, this paper proposes the road paving ways and builds piezoelectric harvesting energy model under vehicle loads. Effect of loads on piezoelectric harvesting properties will then be simulated and analyzed. Some conclusions will finally be drawn.

\section{Layout of the Piezoelectric Harvesting Unit}

To efficiently harvest vibration energy from pavement on large scale and do not affect the vehicle's safety and comfort, the piezoelectric harvesting unit (PHU) proposed in Ref. [6] [7] is adopted here. That is, a piezoelectric vibrator is made up a cylinder disk of $ø 30 \times 10 \mathrm{~mm}$ and copper electrodes welded on up and down surfaces. A 8 $\times 8$ layout of the piezoelectric vibrators in electrically parallel is arranged to constitute a $280 \times 280 \times 20 \mathrm{~mm}$ square of PHU. The space between the vibrators is $4 \mathrm{~mm}$.

Taking the typical pavement structure in China into consideration, the surface of asphalt pavement always includes 2 - 3 layers. The thickness of the top layer is $40 \mathrm{~mm}$. For the better comprehensive result, the PHU is embedded $40 \mathrm{~mm}$ under the bottom of the top asphalt layer. About the optimal design for layouting the PHUs is discussed in detail in Ref. [7]. During the process of reforming the pavement, the top asphalt layer of $40 \mathrm{~mm}$ height is firstly whipped, two trenches of $280 \mathrm{~mm}$ width perpendicular to the vehicle's traveling direction, whose space is $2200 \mathrm{~mm}$, are then excavated. PHU is finally put into the trenches. After the asphalt is replaced, the pavement with PHU is finished. Figure 1 shows the schematic diagram of paving PHU. The PHU width of $280 \mathrm{~mm}$ is larger than the standard tire's width $(\leq 240 \mathrm{~mm})$ for completely harvesting energy from the vehicle load. The spacing distance of $2200 \mathrm{~mm}$ is the same as the standard wheelbase. Each PHU is closely layout. All these are to ensure that each PHU is not only able to absorb road vibration energy, but also to avoid a direct impact on PHU from being damaged.

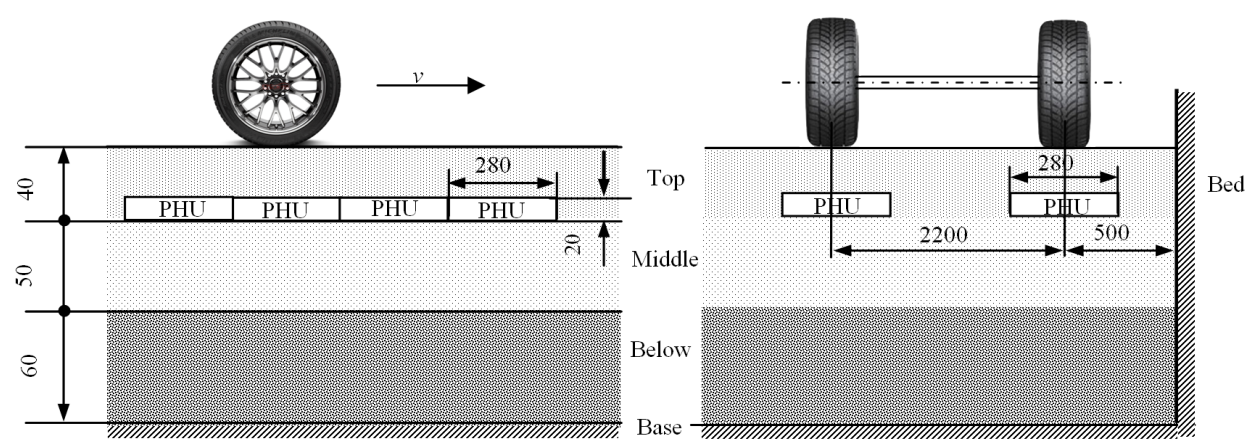

(a)

(b)

Figure 1. Schematic graph of paving PHU under asphalt surface. (a) Longitudinal section; (b) Transverse section. 


\section{Model of PHU under Vehicle Load}

Assuming that vertical load uniformly acts on the pavement when a vehicle passes, the pressure between the tire and the pavement can be deduced from the Meda's empirical formula [8]:

$$
F=0.0042 F^{\prime}+0.29 F_{i}+0.145
$$

where $F$ is the contact pressure in MPa, $F^{\prime}$ is wheel weight in $\mathrm{kN}, F_{i}$ is tire inner pressure in MPa.

Based on Hamilton principle, the pavement's dynamic finite element equation is

$$
[M] \cdot\{\ddot{z}\}+[C] \cdot\{\dot{z}\}+[K] \cdot\{z\}=\{F\}
$$

where $[M],[C],[K]$ are pavement quality matrix, damping and stiffness matrices, respectively. $\{\ddot{z}\},\{\dot{z}\}$ and $\{z\}$ are the node's accelerator, speed and displacement vectors.

The damping matrix of $[C]$ is usually calculated according to the Raleigh damping assumption in the following equation

$$
C=\alpha M+\beta K
$$

where $\alpha, \beta$ are damping co-efficiencies, which are respectively set to be 0.4 and 0.05 based on the empirical value.

When a piezoelectric vibrator is embedded under the pavement, it will generate strain under the vehicle load. The piezoelectric equations are described as the following equations

$$
\begin{gathered}
S_{i}=s_{i j}^{E} T_{j}+d_{m i} E_{i} \\
D_{m}=d_{m i} T_{i}+\varepsilon_{m k}^{T} E_{k}
\end{gathered}
$$

where, $i, j=1,2,3, \cdots, 6 ; m, k=1,2,3 ; S$ is the strain tensor; $T$ is the stress tensor; $s^{E}$ is the compliance tensor of constant electric field condition; $E$ is the external electric field; $D$ is the charge displacement tensor; $d$ is piezoelectric strain constant tensor; $\varepsilon^{T}$ (permittivity) is dielectric constant tensor measure at constant stress condition.

The system, which is constituted by vehicle, pavement and PHU, is a complex multi-bodies dynamics one. To meet the requirement of large stress, low frequency and random vibration and to improve the usage life, the designed PHU adopts the $d_{33}$ mode. According to the above Equations (1), (2), the pavement node displacement $\{z\}$ is obtained. $\{z\}$ is then used as the boundary condition of the system to the node of $E_{i}$ based on Equations (4), (5). Thus, the excited voltage of one vibrator is calculated by Equation (6)

$$
U_{3}=\int E_{3} \mathrm{~d} t=\sum_{i=1}^{6} \int g_{3 i} T_{i} \mathrm{~d} t
$$

where $U_{3}$ is the electric potential (voltage) at the direction 3 caused by polarization changing, which can be obtained via the finite element analysis (FEA); $t$ is the thickness of PZT disk. $g_{3 i}$ is piezoelectric voltage constant, which is deduced from the following equation

$$
g_{3 i}=\frac{d_{3 i}}{\varepsilon_{33}^{T} \varepsilon_{0}}
$$

where, $\varepsilon_{0}$ is vacuum dielectric constant, $\varepsilon_{0}=8.854 \times 10^{-12} \mathrm{~F} / \mathrm{m}$.

The electric energy stored in one piece of piezoelectric vibrator can be calculated by

$$
P_{1}=\frac{1}{2} U_{3}^{2} \frac{\varepsilon_{33}^{T} \varepsilon_{0} A}{t_{p}}
$$

where, $P_{1}$ is the stored electric energy of a vibrator; A is the surface area of PZT disk.

As described above, a PHU, which is the $8 \times 8$ array of the vibrator, can then harvest the total electric energy

$$
P_{2}=64 * P_{1}
$$

where, $P_{2}$ is electric energy harvested by one piece of PHU.

According to the above proposed PHU size and laying way, about 14, 280 pieces of PHU are embedded under 
one mile of two-side pavement. Thus, one piece of PHU may be harvested minimal energy, but the total electric energy is still considerable if the PHU is paved on large scale. Therefore, study on the technology of piezoelectric harvesting energy from pavement vibration will cause a major contribution to social and environment, especially to some countries which mainly rely on coal power generation. The resulting low carbon effect is immeasurable.

It can be drawn from the Formula (1)-(9) that the harvested electric energy of a PHU is relevant to the properties of piezoelectric material and the wheel weight and tire pressure. Here, the effect of wheel weight and tire pressure on the harvested electric energy is discussed.

\section{The Finite Element Model and Material Parameters}

The typical semi-rigid pavement structure is adopted to be the pavement model. The material's parameters of pavement layer and steel are shown in Table 1. Because the contact area between tire and pavement is near a rectangle, tire weight can be treated to evenly act on this area. Table 2 shows the relationship among wheel weight, tire pressure and tire width. The tire contact pressure is also calculated according to Equation (1), as shown in Table 2. PZT-5A material parameters are shown in Table 3. Here, it is supposed that only one tire load is considered and the pressure contact width is the same as the tire's width. Meanwhile, in order to put more attention on the PHU, only the asphalt layer is considered in FEM and the layers of asphalt surface possess the same properties. In viewing to axial symmetry, the finite element model of the pavement piezoelectric harvesting system is built in Figure 2 via ANSYS 10.0.

During simulation, Plane13 is chosen to mesh PZT disk and Plane42 is used to mesh pavement and steel. The electrical potential of the top layer of PZT disk is set to zero, the same potential is set for the bottom of PZT disk. The displacement of the bottom of the model is set to zero. These boundary conditions are shown in Figure 2.

\section{Simulation Results and Analysis}

\subsection{The Excited Voltage of PHU}

Effect of the contact pressure on the excited voltage is shown in Figure 3. It can be concluded that the excited voltage is linear with the contact pressure. The relationship can be expressed as following

$$
U_{3}=0.523+1123.255 * F
$$

From the equation, it can be known that intercept and slope are 0.523 and 1123.255 , respectively. It shows that the fitted line is almost through the origin and the contact pressure takes great influence on the excited voltage.

Table 1. Asphalt and steel material parameters.

\begin{tabular}{cccc}
\hline Material & Elastic modulus/MPa & Poisson ratio & Density $/ \mathrm{kg} \cdot \mathrm{m}^{-3}$ \\
\hline Asphalt & 1800 & 0.32 & 2400 \\
Metal & 200,000 & 0.3 & 7800
\end{tabular}

Table 2. Tire's weight and pressure and width [9].

\begin{tabular}{ccccc}
\hline No. & $\begin{array}{c}\text { Tires' weight } \\
F^{\prime} / \mathrm{kN}\end{array}$ & $\begin{array}{c}\text { Tire pressure } \\
F_{i} / \mathrm{MPa}\end{array}$ & Tire width/mm & $\begin{array}{c}\text { Contact pressure } \\
\text { F/MPa }\end{array}$ \\
\hline 1 & 20 & 0.6 & 220 & 0.4 \\
2 & 40 & 0.7 & 220 & 0.52 \\
3 & 50 & 0.75 & 220 & 0.573 \\
4 & 60 & 0.8 & 220 & 0.63 \\
5 & 80 & 0.9 & 240 & 0.74 \\
6 & 100 & 1.0 & 240 & 0.855 \\
\hline
\end{tabular}


Table 3. PZT-5A material parameters.

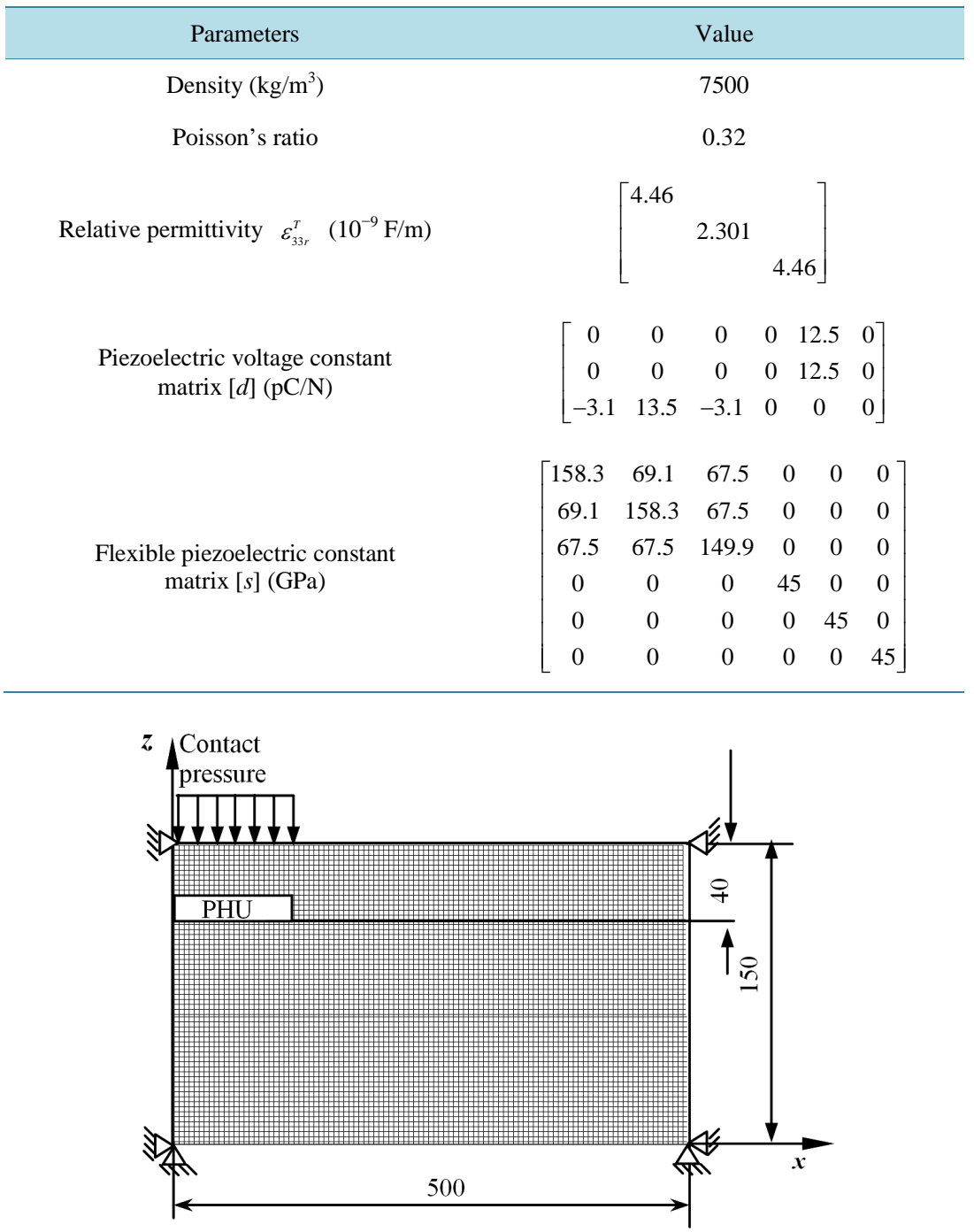

Figure 2. Finite element model.

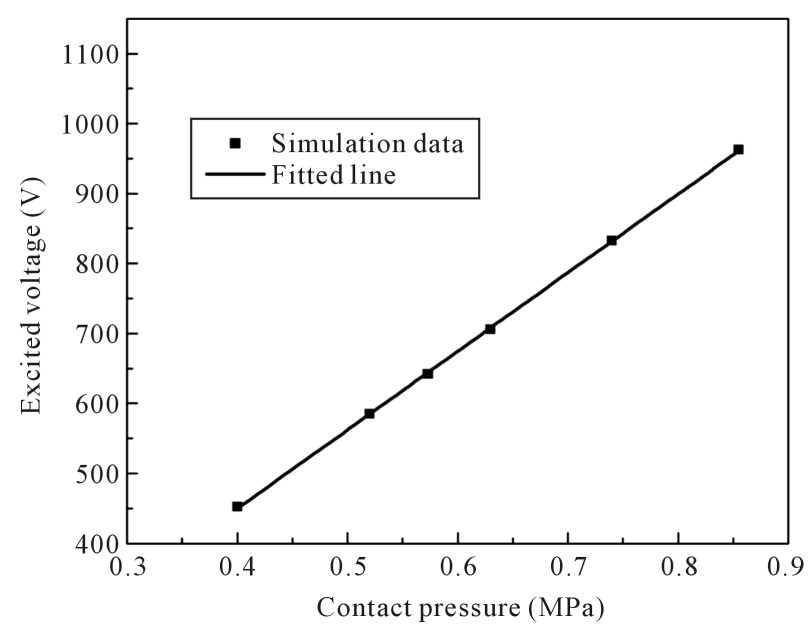

Figure 3. Excited voltage vary with contact pressure. 
The fitted error between the simulation data and the fitted ones is shown in Table 4. The Adj. R-Square is 0.99985 , which is almost near to 1 . It proves that the fitted result of the excited voltage via contact pressure fits very well with the simulation data.

\subsection{The Harvested Electrical Energy of PHU}

Figure 4 shows the harvested electrical energy with one piece of the proposed PHU. It can be seen that at least $100 \mathrm{~mJ}$ of the electric energy is collected with PHU. The relationship of the harvested electrical energy and the contact pressure is expressed as the following exponential equation

$$
P_{2}=0.69773 * \exp (F / 0.58519)+0.00114
$$

According to the fitted statistics, the error and adj. R-square are listed in Table 5. The fitted exponential curve fits very well with the simulation data.

Expanding the project to a length of one kilometer along two lanes, about $450 \mathrm{KWh}$ electrical energy can be harvested with these PHU modules, provided that approximately 600 heavy trucks travel along through the interval per hour on average.

\subsection{The Maximum Deformation of the Pavement}

Figure 5 shows relationship of the maximum deformation of pavement with contact pressure. The relationship can be expressed as a liner equation as follows

$$
z * 10^{-4}=0.00114+0.69773 * F
$$

The error is also calculated, as shown in Table 6. The Adj. R-square is 0.99995. It indicates that the fitted result of the maximum deformation of pavement via contact pressure fits very well with the simulation data.

Taken these comprehensive factors into consideration, when the contact pressure increases, the harvested electrical energy increases. Meanwhile, the pavement displacement also increases. The maximum deformation of pavement is located on the model of $x=0$, the point of the contact center of the tire and the pavement.

\subsection{The Maximum Stress}

Figure 6 shows the stress distribution on the piezoelectric harvesting system from pavement. It can be seen that the maximum stress is located on PZT disk. The maximum stress changes with the contact pressure, as shown in

Table 4. The error of the fitted voltage line.

\begin{tabular}{ccccc}
\hline & Intercept & & Slope & Statistics \\
\hline Value & Error & Value & Error & Adj. R-Square \\
\hline 0.523 & 3.92849 & 1123.255 & 6.16763 \\
\hline
\end{tabular}

\begin{tabular}{|c|c|c|c|c|c|c|}
\hline \multicolumn{2}{|c|}{$y_{0}$} & \multicolumn{2}{|c|}{$A_{1}$} & \multicolumn{2}{|c|}{$t_{1}$} & \multirow{2}{*}{$\begin{array}{c}\text { Statistics } \\
\text { Adj. R-Square }\end{array}$} \\
\hline Value & Error & Value & Error & Value & Error & \\
\hline 0.00114 & 0.00136 & 0.69773 & 0.00213 & 0.58519 & 0.03272 & 0.99978 \\
\hline
\end{tabular}

Table 5. The error of the fitted electrical energy.

Table 6. The error of the fitted deformation line.

\begin{tabular}{cccccc}
\hline & Intercept & & & Slope & Statistics \\
\hline Value & Error & Value & Error & Adj. R-Square \\
\hline 0.00114 & 0.00136 & 0.69773 & 0.00213 & 0.99995 \\
\hline
\end{tabular}




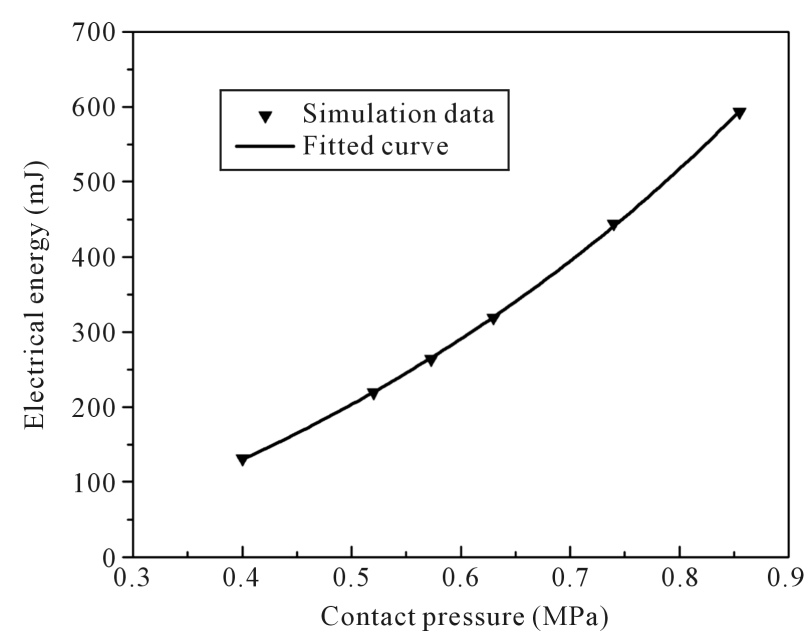

Figure 4. Electric energy vary with contact pressure.

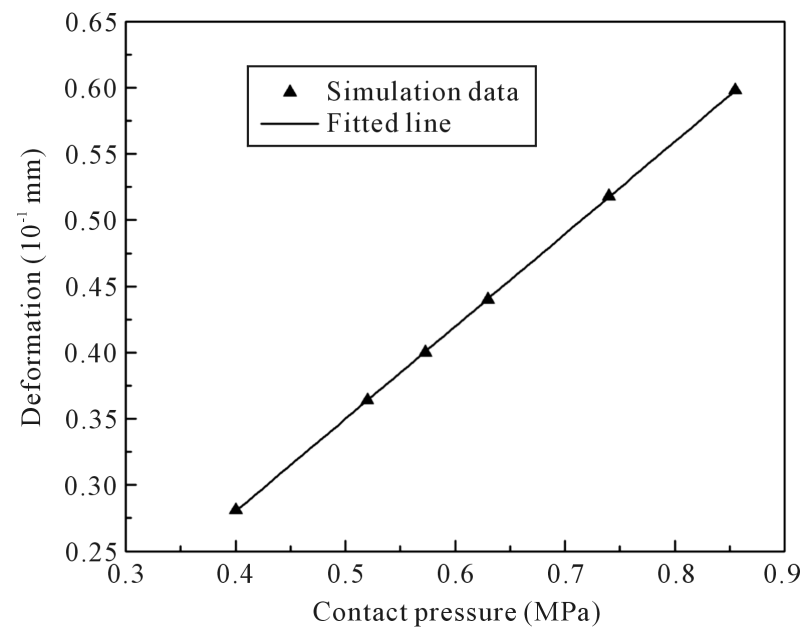

Figure 5. Deformation vary with contact pressure.

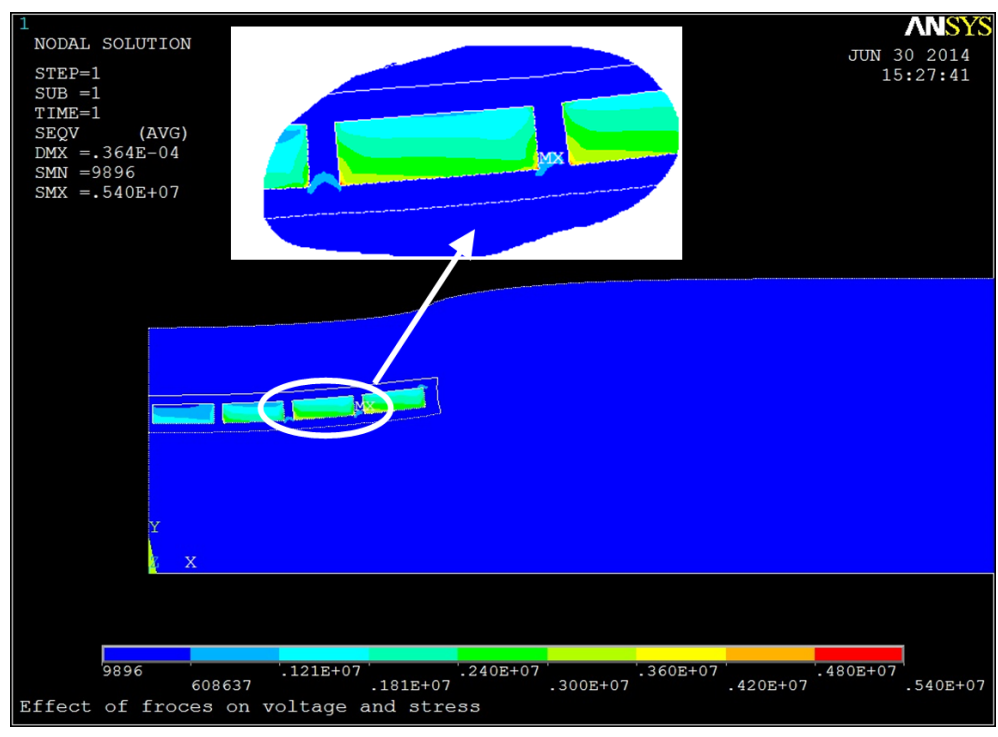

Figure 6. Stress distribution. 
Figure 7. The variation of stress linearly changes with the contact pressure.

The maximum stress on a PZT disk is 8.8 MPa when the maximum contact pressure acts on the pavement. Contrast to the allowable limit stress of $100 \mathrm{MPa}$, 8.8 MPa is so small that the PHU can normally work.

\subsection{Effect of Contact Pressure Frequency}

When a vehicle is driven at different speed, the frequency of the vehicle load varies. Taken a vehicle with 2 axes and the axial distance of $4.5 \mathrm{~m}$, two tire weight of $80 \mathrm{kN}$ for example, the various speeds cause different frequencies of the vehicle load, as shown in Table 7. According to the transit analysis via FEM, the excited voltage also changes with the speed. The simulation result is shown in Table 7.

The excited voltage increases with the increase of the vehicle load frequency. However, its effect is significantly smaller than contact pressure.

Figure 8 shows real-time change characteristics of the excited voltage at $80 \mathrm{~km} / \mathrm{h}$ speed. Obviously, the frequency of excited voltage is the same as the frequency of the vehicle load.

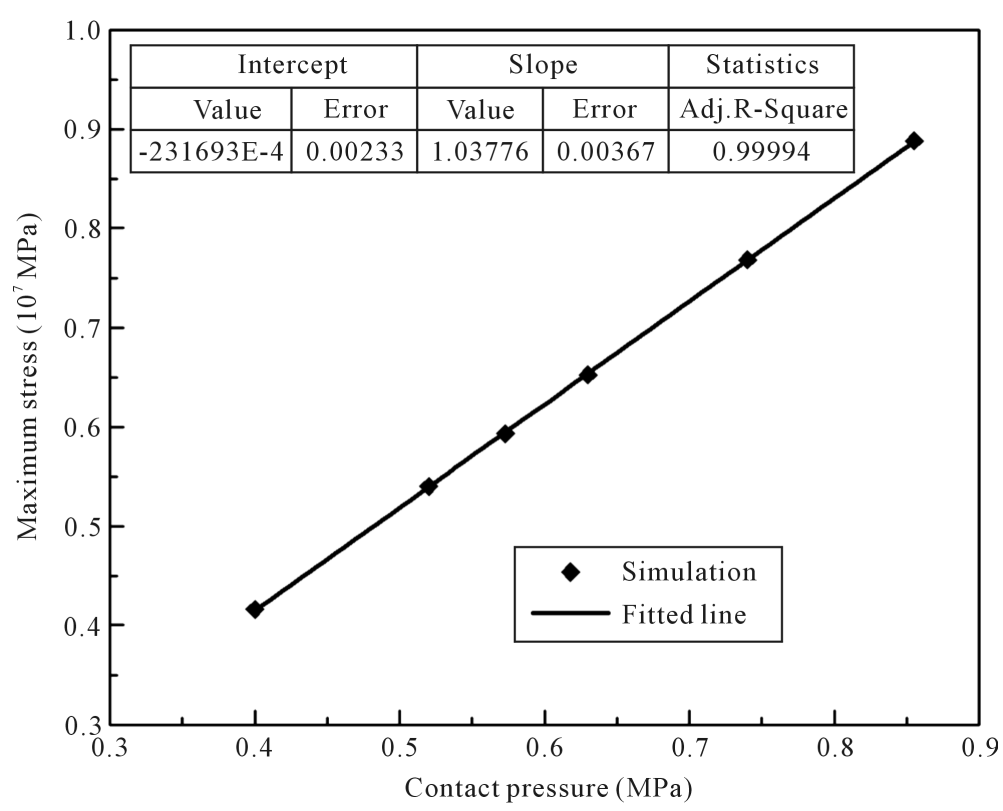

Figure 7. Stress vary with contact pressure.

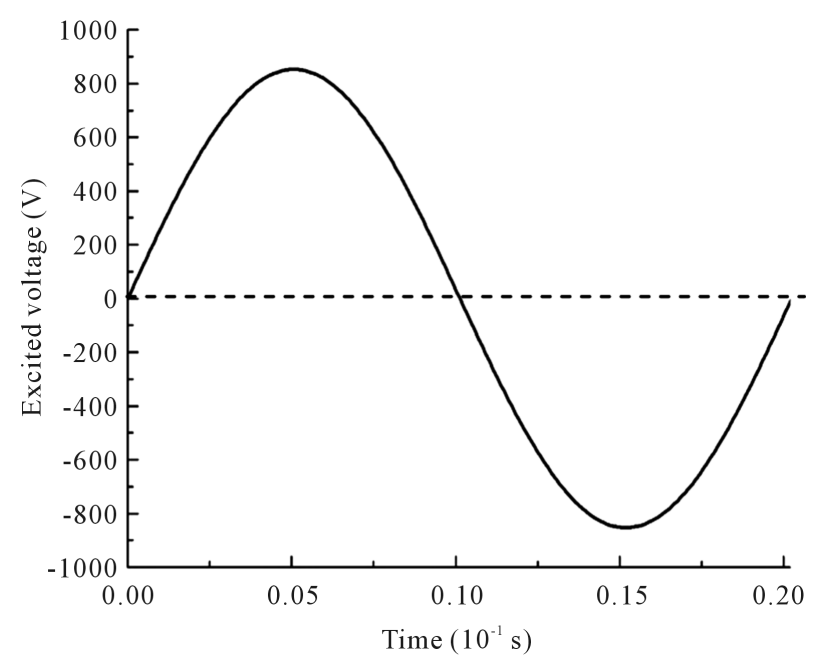

Figure 8. Real-time vary of the excited voltage. 
Table 7. Excited voltages under different speeds.

\begin{tabular}{ccc}
\hline Speed $(\mathrm{km} / \mathrm{h})$ & Frequency $(\mathrm{Hz})$ & Excited voltage $(\mathrm{V})$ \\
60 & 3.71 & 847.6 \\
70 & 4.32 & 850 \\
80 & 4.94 & 852.6 \\
90 & 5.56 & 855 \\
100 & 6.18 & 857.5 \\
110 & 6.79 & 860 \\
120 & 7.41 & 862.4 \\
\hline
\end{tabular}

\section{Conclusions}

The layout of piezoelectric harvesting energy units is discussed. Effect of vehicle loads on piezoelectric energy properties is discussed via the finite element analysis. The harvested energy variation with contact pressure is concluded.

The results show that the maximum stress happens on the PZT disk and lies within the allowable limit stress. Effect of vehicle speed on the excited voltage and the harvested electrical energy is significantly smaller than contact pressure. One piece of PHU can harvest at least $100 \mathrm{~mJ}$ electrical power.

All proves that the technology of piezoelectric harvesting energy from pavement vibration has a promising prospect. The future work will focus on the consistency of PHUs and pavement along with the pavement test.

\section{Acknowledgements}

This research was supported by the National Natural Science Foundation of China (No. 51175359) and the 4th “333 Engineering” Research Funding Project of Jiangsu Province (BRA2014086).

\section{References}

[1] Leland, E.S., Lai, E.M. and Wright, P.K. (2004) A Self-Powered Wireless Sensor for Indoor Environmental Monitoring. WNCG Conference, Austin.

[2] Arms, S., Townsend, C.P., et al. (2005) Power Management for Energy Harvesting Wireless. Smart Structures and Materials 2005: Smart Electronic, MEMS, BioMEMS, and Nanotechnology, Proc of SPIE 2005, 5763, 267-275. http://dx.doi.org/10.1117/12.600302

[3] http://www.innowattech.co.il/index.aspx

[4] Cao, B.-G., Gao, Z.-H., Song, Z.-P., et al. (2005) Method and System of Highway Harvesting Energy from Piezoelectric Vibration: China, 1633009A.

[5] Zhao, H.D., Yu, J. and Ling, J.M. (2010) Finite Element Analysis of Cymbal Piezoelectric Transducers for Harvesting Energy from Asphalt Pavement. Journal of the Ceramic Society of Japan, 118, 909-915. http://dx.doi.org/10.2109/jcersj2.118.909

[6] Sun, C.-H., Du, J.-H., Wang, H.-B. and Shang, G.-Q. (2013) Properties Analysis of Piezoelectric Harvesters from Pavement Vibration. Piezoelectics \& Acoustooptics, 35, 556-560.

[7] Sun, C.-H., Shang, G.-Q., Zhang, Y.-K. and Du, J.-H. (2013) Designing Piezoelectric Harvesting Unit from Road Vibration. Advanced Materials Research, 712-715, 1368-1371.

[8] Shan, J.-S., Huang, X.-M. and Liao, G.-Y. (2007) Dynamic Response Analysis of Pavement Structure under Moving Load. Journal of Highway and Transportation Research and Development, 24, 10-13.

[9] Shi, C.-X., Yang, Q. and Guo, Z.-Y. (2008) Research on Mechanical Properties of Asphalt Pavement in Highway Tunnel. Journal of Highway and Transportion Research and Development, 25, 8-11. 